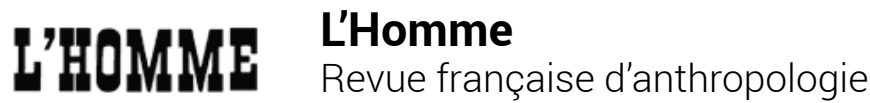

185-186 | 2008

L'anthropologue et le contemporain : autour de Marc Augé

\section{Olivier Maligne, Les Nouveaux Indiens. Une ethnographie du mouvement indianophile Laval, Presses de l'Université de Laval, 2006, 283 p., bibl.}

\section{Marc Lenaerts}

\section{OpenEdition \\ Journals}

\section{Édition électronique}

URL : http://journals.openedition.org/lhomme/18392

DOI : 10.4000/lhomme.18392

ISSN : 1953-8103

\section{Éditeur}

Éditions de l'EHESS

\section{Édition imprimée}

Date de publication : 1 janvier 2008

Pagination : 514-516

ISSN : 0439-4216

Référence électronique

Marc Lenaerts, "Olivier Maligne, Les Nouveaux Indiens. Une ethnographie du mouvement indianophile», L'Homme [En ligne], 185-186 | 2008, mis en ligne le 20 mai 2008, consulté le 24 septembre 2020. URL http://journals.openedition.org/lhomme/18392 ; DOI : https://doi.org/10.4000//homme.18392 


\title{
Olivier Maligne, Les Nouveaux Indiens. Une ethnographie du mouvement indianophile
}

\author{
Laval, Presses de l'Université de Laval, 2006, 283 p., bibl.
}

\section{Marc Lenaerts}

1 Depuis des siècles, l'Amérindien est l'une des figures les plus emblématiques de l'altérité, et une très abondante littérature a déjà été consacrée aux discours dont il a fait l'objet. Mais les réappropriations et manipulations symboliques dont il s'agit ici présentent une dimension particulière, dont l'étude est bien moins fréquente : elles passent avant tout par une mise en actes, dans laquelle l'expérience physique et l'implication personnelle jouent un rôle primordial.

Olivier Maligne consacre ainsi l'essentiel de son livre, Les Nouveaux Indiens, à faire l'ethnographie encore inédite des "indianistes » ou «indianophiles» français - ces gens qui passent tout ou partie de leur temps à "vivre à l'indienne", ce qui peut d'ailleurs prendre des formes assez diverses.

3 Mais les hasards de l'édition ont fait que presque au même moment est paru le brillant ouvrage de Jacques Galinier et Antoinette Molinié, Les néo-Indiens. Une religion du III millénaire ${ }^{1}$. Les auteurs y font une fine analyse de la "déferlante néo-indienne » en Amérique latine, telle qu'elle se donne à voir par exemple dans le culte solaire annuel «néo-inca » de Cuzco ou dans les immenses cérémonies organisées devant le Templo Mayor et sur le Zocalo de Mexico. Là aussi, il s'agit de mise en scène et en actes d'un passé indien en grande partie fantasmé, mais sous la forme nettement plus impressionnante d'un phénomène émergent, qui arrive à mobiliser des dizaines de milliers de personnes issues des milieux les plus divers, et qui est directement lié à la culture New Age, à des revendications d'identité nationale et à des enjeux de pouvoir à tous niveaux.

4 Cette coïncidence pourrait être considérée comme une véritable malchance pour le livre d'Olivier Maligne. Quant à moi, j'aurais plutôt l'impression inverse. En fait, l'inévitable rapprochement met bien en lumière certaines particularités du petit monde 
qu'il décrit, minoritaire, périphérique, utopiste et apparemment un peu replié sur ses activités de routine.

Plutôt qu'un groupe social à proprement parler, les indianophiles français forment un réseau aux limites imprécises, travaillé par toute une série de divergences et de rejets mutuels. Ils partagent néanmoins un certain nombre de représentations et de postulats, dont le moindre n'est pas «qu'il est possible d'actualiser l'univers indien, d'en faire l'expérience hors du monde amérindien » (p. 256).

6 C'est évidemment une position paradoxale, qui peut facilement se révéler inconfortable. Elle soulève immédiatement deux questions, qui préoccupent visiblement beaucoup les indianophiles eux-mêmes: comment faire sien ce qui ne relève pas de soi ? Quel rapport « l'univers indien » que l'on actualise ainsi entretient-il avec la « réalité »?

7 Le problème est d'autant plus ardu que la démarche indianophile se fonde avant tout sur des réélaborations mythiques ${ }^{2}$ de données essentiellement inactuelles, comme le montre le choix des modèles de référence : les périodes de prédilection sont le xvIII ${ }^{\mathrm{e}}$ et le $\mathrm{xIX}^{\mathrm{e}}$ siècles, et certains groupes ethniques comme les Indiens des Plaines (Sioux, Cheyennes, Crows...) y sont tout à fait surreprésentés (p. 47). L'auteur souligne d'ailleurs au passage un paradoxe assez révélateur: le contact direct avec des Amérindiens en chair et en os est en principe toujours valorisé, mais en réalité, bien peu d'indianophiles le pratiquent de façon régulière, et lorsque la rencontre a lieu, elle peut « entraîner un changement d'orientation radical, voire tout simplement l'abandon de l'indianophilie » (pp. 51-52).

8 En fondant leurs pratiques sur de perpétuels décalages avec la réalité (ou mieux, entre différents niveaux d'actualité et d'inactualité), les indianophiles bâtissent donc un monde extrêmement fragile. Mais le plus intéressant se trouve sans doute dans les parades qu'ils inventent contre cette fragilité.

Elles sont fort bien décrites dans la deuxième partie du livre, où l'auteur dresse le panorama des différents courants et des divers " régimes de l'indianité », et les illustre par une série de cas individuels. Il serait trop long d'en faire ici le détail. Disons simplement que cela va du hobbyist qui agit selon le "régime de la tradition » et consacre ses loisirs à imiter minutieusement des objets d'une culture et d'une époque bien précises (Olivier Maligne ne cache pas son admiration pour l'érudition de certains d'entre eux), objets dont il se parera lors de grands rassemblements annuels, jusqu'à «l'utopiste» qui vit en permanence "au tipi», bien plus soucieux de redécouvrir «l'Esprit » amérindien que de se conformer à une tradition précise, en passant par divers types de "professionnels ", dont beaucoup minimisent les aspects économiques de leurs pratiques et préfèrent insister sur leurs rôles de pédagogue et de médiateur vis-à-vis du grand public.

Il y a pourtant des points communs entre ces figures assez disparates. Le plus évident est un souci permanent d'authenticité et de légitimité. Les indianophiles passent leur temps à "préciser ce qu'ils ne sont pas: des gourous ou des mystiques sectaires, des inadaptés sociaux, des névrosés, des passéistes, etc.» (p.92). Le statut réel de leurs pratiques reste toujours problématique, aussi bien vis-à-vis des regards extérieurs qu'au sein même du mouvement: de l'extérieur, on ironise souvent en disant des indianophiles qu'ils «jouent aux indiens », ce dont tous se défendent âprement; mais 
c'est exactement le même reproche que se renvoient sans cesse les différents groupes et sous-groupes.

11 Par ailleurs, c'est toujours sur le mode du faire et dans le temps de l'action que s'objective et se résout (provisoirement) la question de l'actualité ou de l'inactualité de l'univers indien que l'on tente de reconstituer : s'il s'incarne quelque part, c'est dans les gestes et l'expérience vécue d'une fabrication artisanale, de la vie quotidienne «au tipi» ou d'une danse "authentique» - car bien entendu, dans ce domaine des réalisations objectives, l'authenticité demeure également au centre des préoccupations, comme seul critère de légitimité.

On voit comment la combinaison de ces deux aspects peut bloquer l'apparition de reformulations comme celles que décrivent Jacques Galinier et Antoinette Molinié. Maîtres bricoleurs tant qu'il s'agit d'objets, les indianophiles français sont pourtant incapables de se lancer dans un bricolage conceptuel tel que l'entend Claude LéviStrauss: les pré-contraintes symboliques sont pour eux tellement fortes qu'elles semblent empêcher les matériaux d'entrer dans la moindre combinaison nouvelle.

Un dernier aspect tient peut-être aux remodelages que les cultures amérindiennes ont elles-mêmes subies, et qui facilitent leur appropriation par des gens qui n'ont au départ rien de commun avec elles. Olivier Maligne termine en effet son ouvrage par un petit aperçu de la situation québécoise. Il y remarque entre autres que les traditions culturelles amérindiennes y ont été reformulées dans un sens plus normatif. Plus que les contenus, récupérés à partir de données diverses (notamment ethnologiques), c'est le cadre qui a changé, devenant beaucoup plus proche des normes européennes. Quant à savoir si ce type de reformulations fera aussi l'affaire des Amérindiens eux-mêmes, comme le souligne l'auteur lui-même, c'est une question qui reste ouverte.

\section{NOTES}

1. Paris, Odile Jacob, 2006.

2. La mise en place théorique, parfois un peu laborieuse, fait ici référence à l'approche de Roland Barthes, qui intéresse l'auteur par son insistance sur les aspects performatifs du mythe. 


\section{AUTEUR}

\section{MARC LENAERTS}

CNRS-Université libre de Bruxelles, Équipe de recherche en ethnologie amérindienne, Villejuif.

Marc.Lenaerts@ulb.ac.be 